\title{
FUNDAMENTALS OF COMMUNICATION TECHNOLOGY AND NETWORKS
}

\section{Arubere Sunday Ibenate ${ }^{1}$ and Okardi Biobele ${ }^{2}$}

Department of Computer Science \& Informatics Federal University Otuoke, Bayelsa State, Nigeria

${ }^{1}$ Email: ibearubere@gmail.com

${ }^{2}$ Email: bokardi2001@gmail.com

\section{Cite this article:}

Arubere S.I., Okardi B.

(2021), Fundamentals of Communication Technology and Networks. British Journal of Computer, Networking and Information Technology 4(1), 1-12. DOI:

10.52589/BJCNIT-

3VORVHIT.

\section{Manuscript History}

Received: 5 May 2021

Accepted: 22 May 2021

Published: 2 June 2021

Copyright $\odot 2020$ The Author(s) This is an Open Access article distributed under the terms of Creative Commons AttributionNonCommercial-NoDerivatives 4.0 International (CC BY-NC-ND 4.0 ), which permits anyone to share, use, reproduce and redistribute in any medium, provided the original author and source are credited.
ABSTRACT: This paper work generally examines the computer network, the basic classification and types of networks, network goals and the benefits a computer network offers to researchers, individuals and organizations. It also pinpoints the graphical arrangement of the computer network, as well as basic building blocks and general overview of data transmission. Data were collected from primary sources where personal interviews were conducted and were analyzed and presented for statistical decision. This research shows the importance of networks in our ever-growing environment. This paper tends to present an overview and an introduction of some of the fundamental components enabling efficient and effective data communication and transmission. For better network efficiency, we need the right Network components, the right network design, and also the right IT expertise in the design and implementation of any network system. Multiple numbers of computers forming a network tend to enhance computing power and increase the productivity of the network in general.

KEYWORDS: Computer Network, Data Transmission, Data Communication, Topology 


\section{INTRODUCTION}

These days, especially in the business environment, every business no matter how small uses a computer to handle its daily transaction to enable quick and accurate recording. Organizations often need several people to impute and process data simultaneously. In order to achieve this, the earlier model of a single computer serving all the organization's computational needs has been replaced by a model such that a number of separate but interconnected computers do the job. This model is known as a computer network.

By linking individual computers over a network, their productivity has been increased enormously (NOUN: 2002). A stand-alone computer has limited use. The only way it can exchange information with other computers is by using removable storage media like floppy diskettes, flash drive etc. One may not have much issue when using a computer at home, but in a large establishment, where different departments are usually sharing a common data source, effective networking is critical.

A distinguishing characteristic of a general computer network is that data can enter or leave at any point and can be processed at any work station. For instance, a printer can be controlled from any word processor at any computer on the network.

\section{Computer Network}

A computer network consists of one or more stand-alone computers that are linked (connected) together for the purpose of:

i). Resource sharing (fax machine, printer, files etc)

ii). Allowing electronic communication

iii). Share software applications like Ms Office etc. (Andrew. S. Tanenbaum: 1996)

Network Protocol: The protocol defines a common set of rules that are used by computers on the network that communicate between hardware and software entities. (Englewood Cliffs :1989) One very popular protocol for LANs is Ethernet; another popular LAN protocol for PCs is the token-ring network.

Network Goals: Before designing any computer network, engineers should ensure that the designed network fulfils the basic network goals.

$>$ Cost reduction sharing hardware and software resources.

$>$ Reliability by having multiple sources of supply to enable back up.

$>$ Provides an efficient means, of transport for Lange amount of data among various location

$>$ Increases productivity by making it easier to share data amongst users.

- Standards and protocols should be supported to allow many types of equipment from different vendors to share the network. 
> Provides central / distributed management and allocation of network resources like host processors, transmission facilities etc.

\section{Classification of Networks}

When classifying networks we consider the transmission technology i.e., whether the network contains switching elements or not, we can consider two types of networks.

1. Broadcast networks

2. Point -to - point or switched network.

Broadcast networks have a single communication channel that is shared by the entire machine on the network. In this type of network, short messages sent by any machine are received by the entire machine on the network. The packet contains an address field that specifies for whom the packet is sent to. All the machines, upon receiving the packet check for the address field, if the packet is intended for itself, it processes it otherwise the packet is ignored.

\section{Point-to-point or switched network.}

This kind of network has many connections between individual pairs of machines. Such that when a packet travels from source to distinction it may have to first visit one or more intermediate machines. It most requires a routing algorithm because multiple routes of different lengths are available. One common example of a switched network is the international dial-up telephone system.

\section{Major Types of Networks}

\section{Local Area Networks (LANs)}

Local area network is a computer network that spans over a small area. They are mostly confined to a single building or a group of buildings within a campus.

LANs usually connect work stations and PCs. Each node or individual computer in a LAN has its own cpu to execute programs and is able to access data and devices anywhere on the LAN(noun:2002). This enables multiple users to share data and available devices such as printers, fax machines etc. so that one user can communicate with another user by sending email and chart messages. The most common LAN is Ethernet for PCs.

\section{Characteristics of LANs}

1. Confined to small areas and connects several devices over a distance of 5 to $10 \mathrm{~km}$.

2. High speed

3. Low error rates

4. Speeds ranging from $10 \mathrm{mbps}$ to $100 \mathrm{mbps}$ and above. 


\section{Metropolitan Area Network (MANs)}

This is the kind of computer network that is designed for a city or town. The term metropolitan Network is often applied to the new high-speed network technologies that distinguish them from wide area and local area networks. These technologies have pushed the carrying capacity of the communication links into the gigabits range. They can be used over larger areas than the LANs.

\section{Wide Area Networks (WANs)}

A network that covers a large geographical area and covers different cities, states and sometimes even countries is called WAN.

This kind of network is characterized by significance propagation delay in message transmission and by high incidence of lost transmission.

Message units called packets are passed from one intermediate mode to another until they arrive at the destination. Performance quality requires effective routing techniques. Transmission speed ranges from 56kbs to $1.54 \mathrm{mbps}$. It is a point-to-point connection network.

\section{Advantages and Disadvantages of Computer Networks}

\section{Advantages}

The following advantages are considered for this paper work.

1. Resource sharing

2. Performance quality

3. Reliability

4. Accessible resources

5. Incremental growth of computing power.

\section{Disadvantages}

1. Initial cost of installation is very high making many organizations shy away from considering it for use.

2. Security issues suggest a serious threat since hackers can gain access to not duly protected firewalls.

3. Down times can be very catastrophic in terms of loss.

\section{Benefits of computer Networks.}

Connecting workstations and personal computers pc at any range is very beneficial to almost every researchers, student, and institution since it provides for: 
1. Cost reduction by sharing hardware and software resources.

2. Provide high reliability by having multiple sources of supply.

3. Provides an efficient means of transport for large volumes of data among various locations (high throughput).

4. Provides inter-process- communication among user and processor.

5. Sends and receives messages at a very fast rate.

\section{Network Nodes}

Any device that is connected to the computer network is regarded as a node. A node is a generalpurpose computer work station, on which one may run network applications to communicate with another on the network or local applications such as word processing or database programmers. However, an intermediate network device such as a router, which helps in transmitting data or a printer, may also be a network node. To connect a node to a network needs some kind of hardware interface device for doing this. This device is usually called a network adaptor. Taking an IBM-compatible personal computer as an example, the network adaptor is called a network interface card (NIC).

\section{Links and Nodes Fundamentals}

When we talk of a network what generally comes to mind is a computer system that is capable of covering and providing signals that span to a large geographical area even up to a continent, in the real sense, connecting two computers together with a cable also form a network. To consider an effective computer network, its hardware building blocks must contain device elements that are called nodes.

\section{Links}

Links are physical media that connect the nodes. Through a link, signals can be transmitted from one node to another. Digital links carry discrete signals like electromagnetic pulses, whereas analogue links carry continuous electromagnetic signals.

\section{Network Adaptor}

An adaptor is a hardware interference device that is used to connect nodes to the network. Taking an IBM compatible, personal computer as an example, the network adaptor is usually called a network interface card (NIC).

The network adaptor is responsible for sending data from the computer memory to the outside network and also receiving data destined for that computer from the outside network.

Data exchanged between nodes are in units of FRAMES. Computer data is composed of binary digits (bits) $0 \mathrm{~s}$ and $1 \mathrm{~s}$, and a frame is a block of bits. Bits are exchanged between network adaptors. 


\section{Fundamentals of Data Transmission}

Depending on the type of application, a link may be full- duplex, half-duplex or simplex. If two streams of data can be transmitted over the link, in opposite directions at the same time, the link is said to be full-duplex.

A practical example is the telephone network.

If a link allows data to be transmitted in both directions, but only in one way at one time, the link is said to be half-duplex. An example of this is the walkie-talkie communication system.

Simple links only allow data flow in one direction: radio set and TV set is an example. (Stallings W et al: 1994)

\section{Data Transmission Signal}

We know that the two major types of signal are analog and digital signals. The manner in which these two types of signal can be transmitted from source to destination is also two approaches that is

\section{Analog data transmission and Digital data transmission}

An analog signal varies constantly in one or more values, these changes in value can be used to represent data. An analog signal is continuous and can be represented by using sine waves. (Brian K. Williams's et.al 2007)

Human voice is an example of an analog signal. It varies in amplitude (volume) and frequency (pitch)

Digital data transmission describes any system based on discontinuous data or events. Computers are digital machines because at their most basic levels they can be described between just two values $\mathrm{O}$ and 1 or off and on.

\section{Basics of Error Detection.}

The Network should ensure complete and accurate delivery of data from source node to destination node. However, many times, data gets corrupted during transmission. Many factors can corrupt or alter the data that leads to an error.

Several types of error may occur during transmission over the network.

$>1-$ bit error

$>$ Burst error

$>$ Lost message (frame).

1 -bit or single bit error means that only one bit is changed in the data during transmission from the source to the destination node. i.e either $\mathrm{O}$ is changed to 1 or 1 is changed to 0

Burst error: burst error means that 2 or more bits of data are altered during transmission from the source to the destination node. 
Parked loss: this is the fraction of packet sent from a measurement agent to a point for which the measurement agent does not receive an acknowledgement from the test point. This includes packets that are not received by the test as well as acknowledgement that are lost before returning to the measurement agent (Ogude ,U.C 2014) computing information system development information and allude research joined (page 41). (Ogude, uc 2014:41).

\section{Routers and Gateways.}

The router is sometimes called a gateway. It is a device which is used to direct data travel direction between networks of different technologies such as Internet, Novell or IBM Token Ring. The special features of routers are:

i. It provides supports for multi - protocol.

ii. It provides multi - interfaces for different networks

iii. It operates in the internet protocol layer.

Routing usually consists of multiple interfaces to form information across an inter network from source to destination. However, the internet is not a network with Homogenous technologies. It is necessary to build source network devices which are able to inter - change data between difference technologies. Routers are designed for this purpose.

\section{Cabling}

There are three types of cable used in today's network. They include Optic fibre cable, coaxial cable, twisted pair cable.

Optic Fiber cable, this is an extremely thin glass strand. The cable consists of an optic fibre called the core, surrounded by a concentrated layer of glass called the cladding, which is in turn covered by an outer plastic jacket so that the light rays cannot escape the cable and thus travel down the cable in a reflective path. The light source used in fiber optics is usually a laser or a light emitting diode (LED). Fiber optics show very high frequency ranges (higher than $20,000 \mathrm{MHz}$ ) because of this high bandwidth, a single fibre optics cable can support over 30,000 telephone lines and can transmit data over $400 \mathrm{mbps}$.

Coaxial cable: these cables have been around since the early 1940s and are used extensively in local area network long distance toll trunks, urban areas, and cable TV. The technology consists of a single central conductor, surrounded by a circular insulation layer, and a conductive shield. Coaxial cables have high bandwidth up to $400 \mathrm{MHz}$ with much higher quality data transmission than the twisted cable.

Twisted Pair: These are used extensively in telephone circuits in buildings and trunks (Amjad Umar: 1993). Several wires are insulated and enclosed in a cable. A twisted pair cable may include up to 3000 wires paired with a bandwidth up to $250 \mathrm{KHz}$. These cables do have better performance than open wire. Twisted pair cables are good for short distance communication

\section{Network Topology.}

Topology refers to the shape of a network, or the network layout. How different nodes in a network are connected to each other with links and how they communicate with each other is 
determined by the network topology.

Topology is either physical or logical. The term network topology refers to the way nodes are arranged and connected with links Together with the physical medium chosen to implement the link. It determines the speed of the network and the communication efficiency. Its selection depends on the geographical environment, the kind of application running on the network and the implementation cost.

There are three types of topologies that are commonly used.

Bus, star and ring topology. Although we also have three topologies otherwise known as hybrid topology, mesh topology and cellular topology. This paper will restrict us to the first three.

\section{Bus Topology.}

In Bus Topology, all devices are connected to a central Cable, called the bus or back bone. The bus Topology connects workstations using a single Cable. Each node or workstation is connected to the next node on a point-to-point basis. All Nodes are connected to a single cable.

In this type of Topology if one node goes faulty, all nodes may be affected. Since all workstations share the same cable for sending and receiving information.

\section{Star Topology}

Star topology uses a central hub through which all components are connected. In a star topology, the central hub is the host computer, at the end of each connection is a terminal. Nodes communicate across the network by passing data through the central hub.

\section{Ring Topology}

In ring topology all devices are connected to one another in a shape of a closed loop, so that each device is connected directly to two other devices, one on either side i.e. the ring topology connects the workstation (node computer) in a closed loop.

Information travels around the ring from one node to the next. Each packet of data on the ring is prefixed by the address of the station to which it is being sent. When a packet of data arrives, the node checks to see if the packet address is the same as its own, if it is, it grabs the data in the packet. If the packet does not belong to it, it sends the data to the next node in the ring. The common implement of this topography is the token-Ring.

\section{Connection Oriented Service}

Connection- oriented services define a way of transmitting data between a sender and a receiver, in which end- to- end connection is established before sending any data. After establishing a connection, a sequence of packets from the source to the destination can be sent one after the other.

All packets to the message are sent from the same connection. When all packets of a message have been delivered, the connection is terminated. 


\section{Internet}

Internet is an acronym for international network, it is a connection of millions of computer networks together around the world. For the purpose of shearing computer resources, by means of the world wide web. Using known communication standard protocols such as TCP/IP (FUO computer lab. Manual).

When the ARPANET and NSFNET were interconnected, the number of networks, machines and users grew exponentially, many regional networks joined up and connections were made across many countries.

The internet is said to have been officially born around 1982 when the different networks (BITNET, EARN etc), agreed on using the TCP/IP protocol as a standard. ( Noun:2002)

\section{Data Collection and Analysis}

Personal interview was conducted in the United Bank of Africa Plc, Warri and data was collected from both junior and senior staff of the bank and the analysis of the data was presented.

We use statistical hypothesis testing to investigate the data collected to ascertain whether or not the use of multiple computers in a network enhances increased productivity and computing power as against the stand alone computer.

Using the Microsoft Excel package we observed the increased in productivity when higher numbers of nodes are connected to the network

\begin{tabular}{|l|l|}
\hline $\begin{array}{l}\text { Column } \\
1\end{array}$ & $\begin{array}{l}\text { Column } \\
\mathbf{2}\end{array}$ \\
\hline Node1 & 5 \\
\hline Node4 & 23 \\
\hline Node30 & 80 \\
\hline Node7 & 30 \\
\hline Node2 & 20 \\
\hline
\end{tabular}

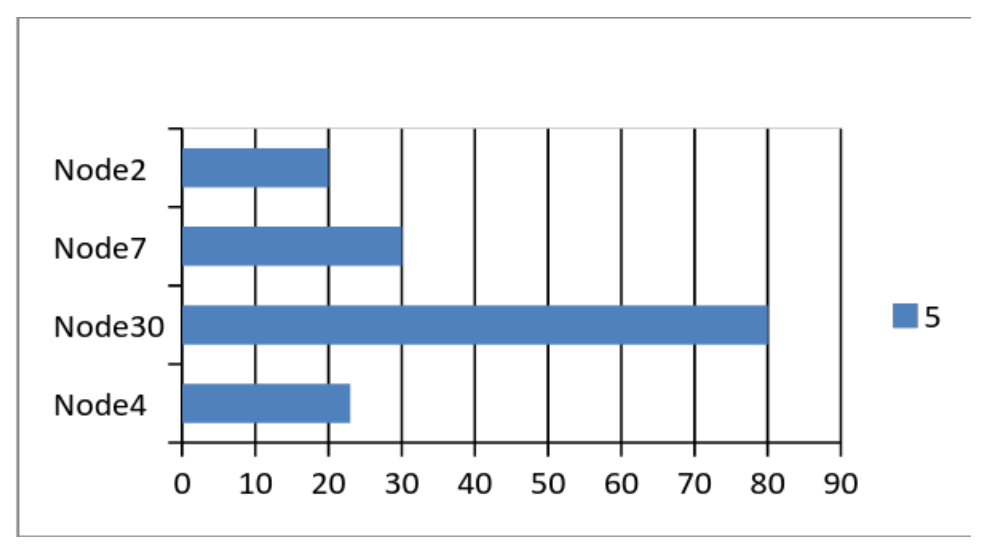




\section{Hypothesis Testing:}

\begin{tabular}{|l|l|l|l|}
\hline Response & Junior staff & Senior staff & Total \\
\hline Agreed & 28 & 7 & 35 \\
\hline Disagreed & 5 & - & 5 \\
\hline Undecided & - & 4 & 4 \\
\hline Row total & 33 & 11 & 44 \\
\hline
\end{tabular}

The use of multiple computers in a network, rather than a single large system eases growth of computing power and enhances productivity?

\begin{tabular}{|l|l|l|l|l|l|}
\hline Response & \multicolumn{2}{|l|}{ Junior staff } & \multicolumn{2}{l|}{ Senior staff } & Total \\
\hline Agreed & 28 & 26.25 & 7 & 8.75 & 35 \\
\hline Disagreed & 5 & 3.75 & - & 1.25 & 5 \\
\hline Undecided & - & 3.0 & 4 & 1.0 & 4 \\
\hline Total & 33 & - & 11 & - & 44 \\
\hline
\end{tabular}

\begin{tabular}{|l|l|l|l|l|}
\hline Fo & Fe & fo-fe & (fo-fe) $^{2}$ & $\frac{(f o-f e) 2}{f e}$ \\
\hline 28 & 26.25 & 1.75 & 3.06 & 0.12 \\
\hline 5 & 3.75 & 1.25 & 1.56 & 0.42 \\
\hline & 3 & -3 & 9 & 3 \\
\hline & 8.75 & -1.25 & 1.56 & 0.19 \\
\hline & 1.25 & -1.25 & 1.56 & 1.25 \\
\hline 4 & 1 & 3 & 9 & 9 \\
\hline
\end{tabular}

\section{Data analysis and computation}

$$
\begin{aligned}
& \mathrm{H}_{\mathrm{o}}, \mu=\mathrm{o} \\
& \mathrm{H}_{1}, \mu \neq \mathrm{o} \\
& \mathrm{D} . \mathrm{F}=\text { degree of freedom }=(\mathrm{c}-1)(\mathrm{R}-1) \\
& \alpha=\text { level of } \mathrm{S} . \mathrm{F}=5 \% \\
& \mathrm{~F}_{\mathrm{o}}=\text { frequency observed } \\
& \mathrm{F}_{\mathrm{e}}=\text { frequency expected } \\
& \mathrm{F}_{\mathrm{e}}=\frac{\text { colume total } * \text { row toatl }}{\text { Grand Total }}
\end{aligned}
$$




$$
\begin{aligned}
& \mathrm{F}_{\mathrm{e}}\left(\mathrm{F}_{\mathrm{o}} 28\right)=\frac{33 * 35}{44}=26.25 \\
& \mathrm{~F}_{\mathrm{e}}\left(\mathrm{F}_{\mathrm{o}} 5\right)=\frac{33 * 5}{44}=3.75 \\
& \mathrm{~F}_{\mathrm{e}}\left(\mathrm{F}_{\mathrm{o}^{-}}\right)=\frac{33 * 4}{44}=3.0 \\
& \mathrm{~F}_{\mathrm{e}}\left(\mathrm{F}_{\mathrm{o}} 7\right)=\frac{11 * 35}{44}=8.75 \\
& \mathrm{~F}_{\mathrm{e}}\left(\mathrm{F}_{\mathrm{o}^{-}}\right)=\frac{11 * 5}{44}=1.25 \\
& \mathrm{~F}_{\mathrm{e}}\left(\mathrm{F}_{\mathrm{o}} 4\right)=\frac{11 * 4}{44}=1.00 \\
& X^{2}=\sum \quad \frac{(f o-f e) 2}{f e}=0.12+0.42+3+0.19+1.25+9=13.95 \\
& \mathrm{D} . \mathrm{F}=\text { degree of freedom }=(\mathrm{c}-1)(\mathrm{R}-1)=(2-1)(3-1)=2 \\
& \alpha=\text { level of } \mathrm{S} . \mathrm{F}=5 \%=0.05
\end{aligned}
$$

Table value $=5.99$

If the calculated value is greater than or equal to table value we reject $\mathrm{H}_{0}$ and accept $\mathrm{H}_{1}$ in this case 13.98 being the calculated as against the table value OF 5.99, we therefore accept $\mathrm{H}_{1}$ which says that multiple numbers of computers forming a network enhances Computing power and increased productivity.

\section{CONCLUSION}

Computer networks are an essential factor in today's business world as well as research institutions, governmental organizations and the world at large. If we must get our job done as quickly as possible, we really do not need to consider distance as a barrier because networking has given us an easy ground for quality data communication and transmission. Taking the advantage that information today is going digital. This paper tends to focus on the fundamentals of communication Technology and Networks.

\section{Revision questions.}

A. Mention three advantages of a computer network.

B. Explain the major differences between Star topology and Bus topology

C. What is a router?

D. Explain the following term
i. 1 bit error
ii. Loss packet
iii. 2 bit error

E. Mention and explain any two network goals. 


\section{REFERENCES}

[1] (A.S Tenenbaum:2002) Computer Networks $4^{\text {th }}$ edition, practice Hall of india New Delhi

[2] William Stalling, Data and computer communication, PHI, New Delhi

[3] (Stalling W. and Van Slyke: 1994:5:124-129) R. Business Data communication (2 ${ }^{\text {nd }}$ edi) New York Mcmillan college publishing company

[4] (Frehlich : 1990-1991) the four volume encyclopedia of Telecommunication

[5] (McGraw Hill:2007) using information Technology a practical introduction to computer \& communication.

[6] (Ogude ,U.C 2014) computing information system development information and allude research joined (page 41). (Ogude, uc 2014:41).

[7] (James Martin:1999) Telecommunication and the computer

[8] William Stallings, Network security Essential- application and standard, person education New Delhi.

[9] http://www.ccs.edu/home/kem/etc/partition

[10] http://www.aciri.org/floyd.tep-mux.htlm

[11] http://www.iec.org/online/tutorials/dwdml 